\title{
DESCUBRIMIENTO DE PITCAIRNIA UNDULATA SCHEIDWEILER (BROMELIACEAE) EN TABASCO, MEXICO '
}

\author{
Sergio Zamudio \\ Coordinación de Ecología del Centro de Investigación y Desarrollo del Estado de \\ Michoacán. Apartado postal 386; 61600 Pátzcuaro, Mich. México
}

\section{RESUMEN}

Se registra por primera vez la presencia de Pitcairnia undulata Scheidweiler en México. La planta se colectó en las laderas calizas del Cerro del Madrigal, cerca de Teapa, Tabasco y se identificó como $P$. undulata, a pesar de las diferencias en el tamaño de algunas estructuras entre los ejemplares de Tabasco y la descripción original de la especie. Se apunta la posibilidad de un error en la asignación de Brasil como localidad de procedencia de las plantas cultivadas en el Jardin Botánico de Berlín, de donde se tomó el ejemplar tipo.

\section{ABSTRACT}

Pitcairnia undulata Scheidweiler is recorded for the first time from Mexico. This plant, collected on calcareous slopes of Cerro del Madrigal near Teapa, state of Tabasco, is identified as $P$. undulata, in spite of differences in the measurements of some structures between the plants collected in Tabasco and the original description. The possibility of a mistake in the designation of Brazil as the site of origin for the plants cultivated at the Botanical Garden of Berlin, on wich the original description was based, is pointed out.

\section{INTRODUCCION}

Durante una excursión de colecta a las laderas calizas del Cerro del Madrigal en las cercanías de la ciudad de Teapa, Tabasco (Fig. 1), el 19 de agosto de 1981 se colectó una Bromeliaceae rupícola de flores rojas vistosas y grandes hojas pecioladas, flexibles y sin espinas (Magaña y Zamudio 340, IBUJAT, IEB, MEXU), que crecía sobre las rocas calizas de los taludes, dentro del bosque tropical perennifolio de Brosimum alicastrum, Poulsenia armata, Pouteria sapota, Pterocarpus hayesii, Aspidosperma megalocarpon, etc., en sitios con clima cálido y precipitación media anual superior a $3000 \mathrm{~mm}$.

Al tratar de identificarla, se encontró que para México no estaba registrada ninguna especie con sus características. Aun cuando los ejemplares coincidieron en general con la descripción de Pitcairnia undulata, citada con duda para Brasil, mostraron diferencias considerables en el tamaño de algunas estructuras (peciolo, lámina de la hoja, brácteas del pedúnculo, etc.). Estas diferencias y la ubicación de la especie en Brasil sugerían la posiblidad de que se tratara de una nueva especie de Pitcairnia, o bien, que fuera un taxon con distribución disyunta,

\footnotetext{
- Trabajo parcialmente subvencionado por el Consejo Nacional de Ciencia y Tecnología, en el marco del Proyecto "Flora Mesoamericana".
} 
Acta Botánica Mexicana (1988), 2:5-9

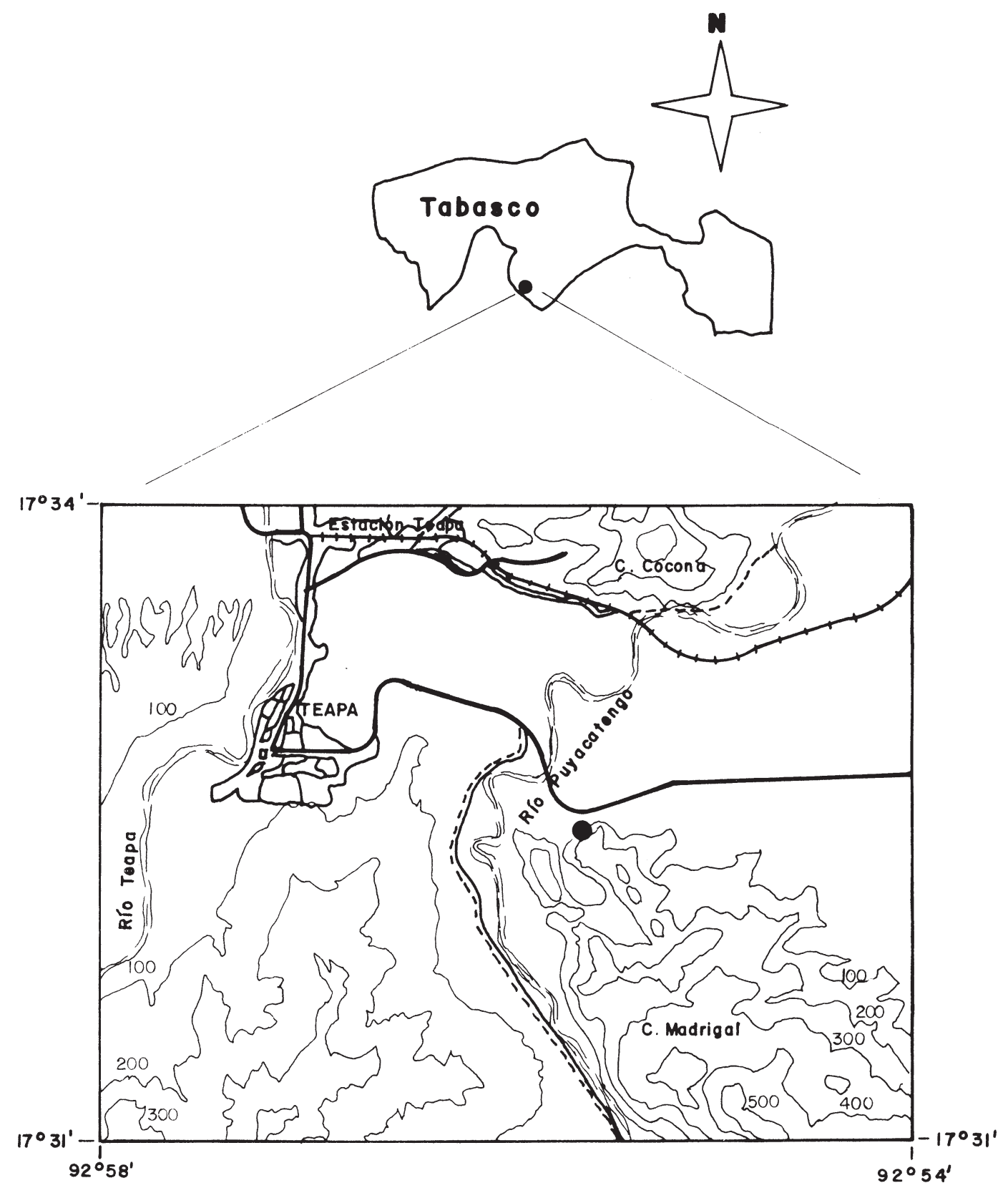

Fig. 1. El punto negro muestra la localidad de colecta de Pitcairnia undulata Scheidweiler en la ladera noroeste del Cerro del Madrigal, al este de Teapa, Tabasco. 
en el que la población mexicana discrepara del tipo sudamericano en algunas características, en concordancia con el aislamiento geográfico.

Por la literatura se sabe que $P$. undulata fue descrita por primera vez en 1842 por Scheidweiler, quien se basó en ejemplares provenientes de plantas cultivadas en el Jardín Botánico de Berlín, a las que se asignó Brasil como localidad de procedencia, sin ninguna referencia más detallada (Mez, 1896, p. 371). Al parecer, desde que esta planta fue colectada y enviada al Jardín Botánico de Berlín no se volvió a encontrar en estado silvestre, por lo que sólo se conoce en cultivo.

Al comparar los ejemplares colectados en Tabasco con fotografías de los ejemplares de Morren s.n., procedentes del Jardín Botánico de Lieja, en Bélgica, depositados en el herbario del Royal Botanic Gardens, en Kew (K), se encontró una fuerte semejanza, coincidiendo sobre todo en la etapa madura de la floración.

En agosto de 1983 se colectaron otros especímenes (Zamudio 968, ENCB, IEB, MEXU), tanto para ejemplares de herbario como para cultivarse en macetas. Al permanecer en condiciones diferentes a las naturales la planta sufrió varios cambios: se retrasó el crecimiento de las hojas nuevas y disminuyó notablemente el tamaño de la hoja y de la inflorescencia, haciéndola coincidir mejor con la descripción de $P$. undulata.

Al revisar con más cuidado las características de los ejemplares colectados y compararlas con la descripción que de esta especie se hace en la Flora Neotrópica, basada en ejemplares provenientes de varios jardines botánicos (Smith \& Downs, 1974, p.372), se encontró que a pesar de que los especímenes de Tabasco son más grandes, existe una fuerte sobreposición en los intervalos de variación del tamaño de las estructuras, con la excepción de los pétalos y las anteras, que resultaron ligeramente más pequeñas en los ejemplares de Tabasco de lo que se señala en la descripción (Cuadro 1).

Cuadro 1. Comparación del tamaño de algunas estructuras de $P$. undulata (según Smith \& Downs, 1974) y de los ejemplares colectados en Tabasco, Méx.

\begin{tabular}{|l|c|c|}
\hline \multicolumn{1}{|c|}{ Caracteristicas } & $\begin{array}{c}\text { P. undulata } \\
\text { (Smitth \& Downs, 1974) }\end{array}$ & $\begin{array}{c}\text { P. undulata } \\
\text { (Ejemplares de Tabasco) }\end{array}$ \\
\hline PECIOLO (largo) & $20-55 \mathrm{~cm}$ & $39-83 \mathrm{~cm}$ \\
\hline LAMINA DE LA HOJA $\begin{array}{c}\text { (largo) } \\
\text { (ancho) }\end{array}$ & $\begin{array}{l}60 \mathrm{~cm} \\
18 \mathrm{~cm}\end{array}$ & $\begin{array}{l}46-77 \mathrm{~cm} \\
14-20 \mathrm{~cm}\end{array}$ \\
\hline BRACTEAS DEL ESCAPO (largo) & $3.5 \mathrm{~cm}$ & $2.1-6.7 \mathrm{~cm}$ \\
\hline INFLORESCENCIA (largo) & $20-40 \mathrm{~cm}$ & $26-62 \mathrm{~cm}$ \\
\hline PEDICELOS (largo) & $0.8-1.5 \mathrm{~cm}$ & $0.4-1.8 \mathrm{~cm}$ \\
\hline SEPALOS (largo) & $2.6-3.0 \mathrm{~cm}$ & $2.0-3.4 \mathrm{~cm}$ \\
\hline PETALOS (largo) & $6-8 \mathrm{~cm}$ & $3.6-5.7 \mathrm{~cm}$ \\
\hline ANTERAS (largo) & $1 \mathrm{~cm}$ & $0.8-0.9 \mathrm{~cm}$ \\
\hline
\end{tabular}




\section{DISCUSION}

La similitud tan grande de las plantas de Tabasco con los ejemplares de Morren, la sobreposición de las medidas ilustradas en el Cuadro 1, así como las modificaciones observadas en cultivo nos permiten afirmar que se trata de una sola especie, cuya variación se desconocía por falta de colectas.

Con respecto a la distribución geográfica de este taxon se considera que no es probable un área disyunta entre Brasil y el sureste de México, sin que la planta aparezca en los bosques tropicales del norte de Sudamérica o en Centroamérica.

El descubrimiento de $P$. undulata en Tabasco y la carencia de colectas de la planta en estado silvestre en Sudamérica, parecen indicar que México y no Brasil es el sitio de origen de esta especie.

Resulta difícil afirmar con precisión quién colectó esta planta y cómo llegó al Jardín Botánico de Berlín antes de 1842 (fecha en que fue publicada la especie), pues no se cuenta con suficiente información al respecto. Sin embargo, ha sido posible obtener algunos datos que nos dan una idea de lo que pudo haber ocurrido.

Hemsley (1886-1888, pp. 125-126), relata que entre 1835 y 1840 se encontraba en México un grupo de exploradores belgas, que bajo los auspicios de su gobierno o de instituciones particulares colectaban plantas y animales para su estudio en Europa. Según Rovirosa (1888, pp. 211-217), dos de estos colectores, Linden y Ghiesbreght estuvieron en Tabasco en varias ocasiones, la primera vez entre 1839 y 1840 y colectaron precisamente en los cerros en que crece $P$. undulata; de acuerdo con el mencionado autor: "En 1839 regresó á Europa Ghiesbreght, y a su vuelta á México en aquel mismo año, visitó con Linden á Tabasco. Ambos viajeros trabajaron activamente en este Estado, especialmente en los alrededores de Teapa y en las sierras de Chiapas, hasta el mes de Marzo de 1840, en que se embarcaron conduciendo preciosas colecciones. Volvieron á Teapa á fines del año referido; pero el grande acopio de plantas y animales que en poco tiempo llegaron á obtener, les hizo sentir la necesidad de dirigirse por tercera vez á ultramar. Cuando su afán por nuestra historia natural les trajo nuevamente a las playas mexicanas, emprendió Ghiesbreght sólo sus correrías por el interior de la República"...

Las plantas vivas que llevaron a Europa durante estos viajes fueron conducidas al principio a Luxemburgo, más tarde a los viveros de Linden en Bruselas y Gante, de donde se distribuyeron ampliamente (Stafleu \& Cowan, 1981, pp. 41-43).

De acuerdo con lo anterior es muy probable que los individuos de $P$. undulata arribaron por única vez a Europa como resultado de estas expediciones y que por la pérdida de su localidad de colecta se consideraron procedentes del Brasil, país en el que Linden y Ghiesbreght estuvieron entre 1835 y 1837.

El caso de $P$. undulata no es único, pues una equivocación semejante ocurrió con $P$. recurvata, especie también descrita por Scheidweiler de ejemplares provenientes de plantas 
cultivadas en el Jardín Botánico de Berlín, la cual fue citada originalmente para Brasil, pero en realidad su distribución conocida abarca el sur de México, Guatemala y Belice (Smith \& Downs, op. cit., p. 376).

\section{CONCLUSIONES}

Con estas evidencias se puede concluir que las plantas colectadas en el Cerro del Madrigal, en las cercanías de Teapa, Tabasco, corresponden a $P$. undulata y que es la primera ocasión en que se colecta la planta en estado silvestre. Este es un nuevo registro para México y probablemente la especie no es de Brasil.

\section{AGRADECIMIENTOS}

Agradezco al Biól. Fernando Guevara-Fefer por sus comentarios, la revisión y la fotografía de los ejemplares depositados en el herbario del Royal Botanic Gardens, Kew; al Dr. J. F. Utley por sus comentarios importantes para definir la especie; al Dr. J. Rzedowski, por la revisión del escrito y sus valiosas sugerencias, y a la Sra. Rosa Elena Murillo por la mecanografía del trabajo.

\section{LITERATURA CITADA}

Mez, C. 1896. Bromeliaceae. In: De Candolle, A. y De Candolle C. Monographiae Phanerogamarum 9: 1 990.

Hemsley, W. B. 1886-1888. Botany, Vol. IV. In: Godwin, F. D. \& O. Salvin. Biologia Centrali-Americana. R. H. Porter. London. $498 \mathrm{pp}$.

Rovirosa, J. N. 1888. Vida y Trabajos del Naturalista Belga Augusto B. Ghiesbreght, Explorador de México. La Naturaleza. Segunda Serie. Tomo I. 1887-1890. 508 pp.

Smith, L. B. \& R. J. Downs. 1974. Pitcairnioideae (Bromeliaceae). Flora Neotropica. Monograph No. 14. Hafner Press. New York. 660 pp.

Stafleu, F. A. \& R. S. Cowan. 1981. Taxonomic Literature. Vol. III. Second Edition. Bohn, Scheltema \& Holkema, Utrecht \& dr. W. Junk b. v. Publishers, The Hague. 980 pp. 